\title{
Harmonization of TIQA Standards for Specialized Texts
}

\section{Roksolana V. Povoroznyuk}

Taras Shevchenko National University of Kyiv, Kyiv, Ukraine

\begin{abstract}
This research explores translation and interpreting quality assessment standards (TIQA), selecting those fit for the purpose of specialized translation quality assurance, with the aim to systematize them into a step-by-step framework, referred to as "the TIQA pyramid", a framework that provides valid and reproducible benchmarks that are endowed with universal features and reflected in codes of ethics and professional standards. The TIQA standards may be subdivided into two major groups: text-oriented and ethicaldeontological ones. Such classification is based on the notion of translation quality which is the projection of a translator (interpreter)'s personality (inchoate quality assurance arising out of a system of ethical and deontological precepts), or of textual requirements (choate quality assurance arising out of a system of textoriented criteria). The "pas-de-trois" in a translated interaction among the commissioner of a specialized translation, its performer and enduser is grounded in the presumably existing mediated communication contract (typically a translation brief). Its positive upshot is manifested in the confidence-imbued multi-party polypragmatic interlingual and intercultural behaviour; the negative, however, is underscored by its implicit nature which leads to the absence of a concerted system of quality criteria, resulting in a lack of satisfaction and mutual trust.
\end{abstract}

Keywords---equivalence, linguistic validity, quality assessment, skills, translating.

\section{Introduction}

Hatim and Mason once memorably wrote that "translating involves a conflict of interests; it is all a question of where one's priorities lie" (Hatim \& Mason, 1990). In this manner, they have neatly delineated the problem of specialized translation/interpretation quality assessment (TIQA), be it a spoken or a written mode; if the language is a valuable skill and a tool of influence at the same time,

Linguistics and Culture Review (c) 2021.

Corresponding author: Povoroznyuk, R.V.; Email: rpovoroznyuk7637-1@tanu.pro

Manuscript submitted: 9 April 202 1, Manuscript revised: 18 June 2021, Accepted for publication: 27 July 2021

678 
who is to evaluate the extent of its efficacy: a user or a client? Who will have the final say? Who will adopt and veto the translatorial quality assurance decisions? To this effect, the Recommendation on the legal protection of Translators and Translations and the practical means to improve the Status of Translators, known as the Nairobi Declaration urges relevant organizations or associations to: promote the adoption of standards governing the translating profession; such standards should stipulate in particular that the translator has a duty to provide a translation of high quality from both the linguistic and stylistic points of view and to guarantee that the translation will be a faithful rendering of the original (UNESCO, 1976).

An intercultural mediator finds himself/herself both at the centre and in the crosshairs of domain-specific, decision-fraught communication as the proud bearer of a linguistic and communicative weapon. It is up to him/her whose side to take: the commissioner or the client, and who to empower of his/her own accord. To the same extent, the notion of translation quality may be viewed as the projection of a translator (interpreter)'s personality (inchoate quality assurance arising out of a system of ethical and deontological precepts) or out of textual requirements (choate quality assurance arising out of a system of text-oriented criteria). However, as it often happens with human intellectual pursuits, the abstract idea arising from the solid instrument, choate quality assurance often resonates with, and even mirrors, the inchoate one. In terms of the text-bound (choate) and ethical-deontological (inchoate) criteria, the parallelism is apparently found in the groups of professional criteria under the umbrella term of professional integrity, and fitness for purpose; the latter doubles as a translation industry-specific concept to ensure the economic viability of any translation quality assurance procedure under the given circumstances, this assumption being proved by our own findings (Diaz et al., 2011; Coban, 2015; Malakoff, 1992). Quality assessment criteria (QAC) for translation and for interpreting would mirror each other with differences in their exact definition and application. However, criteria in TIQA can be regarded and put to practice as a complex hierarchy or a simple checklist, with or without their arrangement in the order of importance in a given situation.

According to the approach selected by the assessor (reviser/reviewer/editor/commissioner), these criteria may be superimposed over one another, blended together, or one specific criterion or a set of criteria would supersede the rest. Consequently, criteria should be adjustable in accordance with the situation. In this respect, it should be noted that the purely theoretical debate about adequacy/equivalence as such, without reference to the operational framework (translation brief, working conditions, etc.), is not conducive to TIQA and its criteria standardisation.

Yet, as the FIT (Fédération Internationale des Traducteurs) Position Paper on International Standards states, "translation is not a commodity, but a creative service that can never be fully standardised in the hope that standardisation alone will guarantee high quality". The conduit model of a mediated specialized (domain-oriented) communication, claiming that an interlingual mediator should act as an invisible code-switcher, has long been discarded by the translation scholars, along with the notion of a translator's neutrality. Nowadays, we tend to 
view the role an interlingual mediator plays as that of the researcher's essential wingman, a co-constructor of the scholarly domain-specific meaning. The metaphorical "pas-de-trois" among the commissioner of a specialized translation, its performer and end-user is grounded in the presumably existing "mediated communication contract", i.e. a set of necessary individual skills for mediated communication on the basis of a limited number of explicit text-bound quality clauses (criteria) (Dresp-Langley, 2009).

The mediated communication contract shapes the mediated communication unfolding and unites under the umbrella of common "horizon of expectations" the quality assumptions and requirements the parties involved in this mediated communicative act may share. The positive upshot of the "mediated communication contract" is the confidence-imbued multi-party polypragmatic interlingual and intercultural behaviour, however limited by the fact of its implicit nature. The consequence of this implicitness is the absence of a concerted system of quality criteria that results in a lack of satisfaction and mutual trust. What's even more important, the parties to the contract are reluctant to complain about the reasons of their frustrations.

Obstacles to the successful mediated specialized communication occur irrespective of the participants' demographic characteristics or the mediated communication domains. The three areas, where participants are most likely to differ, concern:

- ideas about the causes underlying the mediated specialized communication defects;

- expectations of the mediated interaction;

- verbal and non-verbal communication styles (Hudelson et al., 2013).

Very often the TIQA-originating misunderstandings or discrepancies are culturebound. For instance, the Westernized view of a translator's visibility manifests itself in:

- introducing or positioning him/herself as a party to the interpreted event;

- setting communication rules (for example, turn-taking) and controlling the traffic of information;

- paraphrasing or explaining terms or concepts;

- sliding the message up and down the register scale;

- filtering information, etc (Angelelli, 2004).

However, it is completely outside the bounds of some patriarchal communities treating representatives of a certain professional domain (medicine, religion, law etc.) or government office as something close to deity whose statements may be subject to translation; their occupation and status are thus covered in a pall of mystery, enshrined by the rituals, and the translator who is trying to regulate the potentate-graced interaction would certainly be admonished and his/her visibility constrained (Simanjuntak, 2019; Singleton, \& Krause, 2009).

Another impediment to a successful translation quality assurance and assessment is found in the inherent lack of equality underlying the above- 
mentioned "pas-de-trois" of a translated interaction. To a similar extent, the commissioner of a specialized translation and its end-user are crippled by their absence of linguistic common ground; thus, making their requirements as to the translation quality only in a provisional "blueprint" manner. What's crucial, the timeline of their standards being either met or violated also varies: in case of a commissioner, the requirements are pre-formulated (a "translation brief") (Nord, 1997). Before the mediated interaction, and not open to adaptation under the imposed circumstances, while in case of an end-user, the requirements become apparent only via reception, i.e. have a remote effect. This provisional "blueprint" nature of the translation brief is further exemplified in I. Torresi's definition as "the information provided to the translator by the client and/or the agency or other intermediaries about their expectations regarding the target text. the brief should always be respected or negotiated with the final client before handing in the target text. Intentional departures from the brief should always be motivated and offered as an alternative to one or more versions that adhere to it" (Torresi, 2014).

When stating that overall the translator's work would be "ideally governed by a brief from the client", B. Mossop nonetheless claims that "given the users and the use, the client's specified strategy [in the brief] may not be appropriate. The client is not always right" (Mossop, 2014). Despite the provisional and remote nature of the translation brief's quality requirements, they are still casting their shadow over the translatorial decisions of a more immediate procedural nature. For instance, G. Toury's suggested polarity of adequacy and acceptability epitomizes the translator's affinity towards ST (prototext or the commissioner's requirements) or towards TT (metatext or the end-user requirements) (Toury, 1995); in a similar vein, E. Nida speaks of a formal vs. dynamic equivalence, J. House of an overt vs. covert translation, Venuti (1995), of fluency vs. resistance etc. On a more practical level, this polarity of translatorial quality standards manifests itself in the translatorial behavior, and procedures/techniques elected or discarded by the translator. If, for instance, the interactional co-constructor aligns with the speaker of a dominant language, he/she promotes the "gatekeeping", conveying but not providing access to information for the less dominant speakers. By contrast, attention to the recipient's linguistic and sociocultural needs turns into a practice of advocating for the less dominant speakers through cultural brokering.

Based on the above-mentioned considerations, we define the present study's aim as the exploration of TIQA standards, selection of those fit for the purpose of specialized translation quality assurance and provision of a step-by-step framework of criteria promoting the optimal specialized target text in translation.

\section{Materials and Methods}

Faced with the problem of selecting the apt TIQA standards serving the domainoriented translation, we have developed the following multifactor study procedure. At the first stage, we have analysed an array of existing translation studies of a general nature, delving into the issue of translation quality and its underlying criteria. As we have found no exhaustive explorations of specialized TIQA standards, we were then obliged to revert to such general scientific methods, as 
induction, deduction, extrapolation and generalization, trying to decide upon the common ground which all of the translation standards share. In our view, this common ground may be presented as tertium comparationis which, for some scholars, is a piece of reality or thought (a referent, a function, a message) that stands outside all languages. Thus, Danica Seleskovitch recommends "listening to the sense," or "deverbalizing" the source text so that one is only aware of the sense, which can be expressed in all languages (théorie du sens) (Seleskovitch, 2015). To follow Seleskovitch's precept, being charged with a task of rendering a specialized text, the translator goes from the source text to a third element of comparison (a referent, a function, a message), then from this element to the corresponding target text.

Pym (2014), by contrast, considers tertium comparationis to be a bedrock of equivalence, a relation of 'equal value' between a start-text segment and a targettext segment which can be established on any linguistic level, from form to function (Pym, 2014). The author states that, viewed from this perspective, the equivalence is a commercial projection, a certain act of trade (or contractual agreement) revolving around the value of product rendered by the tender. Seconded by E. Gutt, Anthony Pym (2014), articulates an iconoclastic view: the tertium comparationis is an implicit trust of the commissioner and recipient in the performer's veracity and professionalism. In other words, no linguistic comparison of ST (standard text) and TT (translated text) is necessary; the TIQA standards are based on the presumption of equivalence, implicit belief (or "mediated communication contract") by no means to be shattered by the translator's clumsy attempts. At the same time, in connection with the specialized texts in rendering, extrapolation of the generalized TIQA standards led us to Venuti's (1995), widely-appreciated warning that fluency (i.e. normalization of the source via rendering, the cornerstone of "mediated communication contract") results in a parochialism or simplification (vernacularization) of expert-originating discourse.

The similarity of meaningful value is ascertained by semiotic analysis, which may be of a componential or differential character. By its means, we found out that besides semantic, grammatical and idiomatic equivalences functioning across the genres and text types, the specialized texts are graced by experiential and conceptual equivalences, both vital for the cross-cultural adaptation. Experiential equivalence requires that "the situations evoked or depicted in the source version should fit the target cultural context" (Guillemin et al., 1993). Conceptual equivalence refers to "the validity of the concept explored and the events experienced by people in the target culture, since items might be equivalent in semantic meaning but not conceptually equivalent" (Guillemin et al., 1993).

Our findings on equivalence were further supported by observation and distributive analysis. The specialized TIQA standards rely heavily on the correct representation of specialized (domain-centered) concepts, their definitive and thesaurus analysis. Those concepts, that were inadequately highlighted by the ST (due to their implicit connotations) or just entered the TT, required descriptive analysis and contextual study; their application brings forth a specific validity tool making abstract tenets of translation quality studies hands-on and down-toearth. 
The principle of linguistic validity of a translated domain-specific text stipulates that the rendered version plays the same role in the target culture as the original in the primary one. It relies upon a premise that the target audience construes the specialized message in the same way as the original audience did (this view is far from revolutionary: for instance, E. Nida expressed the same idea using the polarity of "formal" vs. "dynamic" equivalence). However, collecting data in different cultures with the aim of obtaining comparative results requires that the specialized text has a cross-cultural validity, i.e., that translation and measurement equivalence are ensured or at least tested (Grunert et al., 1993).

Besides the linguistic and cross-cultural validity, there are other types to be taken into account while developing the TIQA standards for domain-specific discourses. Construct validity is determined by an evidence that relationships among items and concepts conform to a priori assumptions concerning logical relationships. If the results are consistent with a pre-existing hypothesis, it is a convergent validity, otherwise - a discriminant validity. Criterion validity is the extent to which the numerical indicators (units of measurement) are related to a known 'gold standard measure' of the same specialized concept (Chen et al., 2002; Saule \& Aisulu, 2014).

By far the most important type of validity in terms of translation is the content validity, i.e. the extent to which the TIQA standard encompasses the concept of interest. Content validity is specific to the population, conditions, cultural and environmental issues. In its Guidance for Industry, the FDA mentions the content validity among the standards against which the quality of translated version is tested: "Regardless of whether the instrument was developed concurrently in multiple cultures or languages or whether a fully developed instrument was adapted or translated to new cultures or languages, we recommend that sponsors provide evidence that the content validity and other measurement properties are adequately similar between all versions used in the clinical trial. We will review the process used to translate and culturally adapt the instrument for populations that will use them in the trial".

In ascertaining the validity of TIQA standard application we have deliberately waivered the dichotomy of polar qualifiers: "professional/ad hoc", "quality/amateur", "expert/novice", as we consider them inconclusive and deficient in terms of a research study tool array. Our opinion is grounded in the "Standard Guide for Quality Assurance in Translation", which claims: "Quality translation cannot be defined on the premise that there is only one correct, highquality translation for any given source text. there can also be a variety of correct ones, depending on the specifications defined for a given project and the choices made by the individual translator".

We were rather striving to develop a step-by-step framework of standards, referred to as "the TIQA pyramid", with a view that the task of performing a highquality (professional, expert) specialized translation is possible only though achieving valid and reproducible benchmarks at each and every step (Rezvani \& Vakilinejad, 2014; Kashgary, 2011). 


\section{Results and Discussion}

The concept of equivalence lies at the heart of translation methodology and paradoxically creates a dividing line between the classical oppositions: adequacyoriented translation vs. acceptability-oriented translation, overt vs. covert, indirect vs. direct one. Pym distinguishes between 'natural equivalence' existing prior to the translating act and 'directional equivalence' which depends upon the choice that the translator makes among several translation solutions (Pym, 2014). The task of evaluating the quality of a specialized text's translated version appears notoriously difficult, since it is not entirely clear what the focus of evaluation should be. On the one hand, an accurate translation is to convey the original text's meaning, on the other - a fluent delivery is desirable as it should be read easily.

Equivalence requires functional constancy (Funktionkonstanz). In specialized translations, the long-standing debate about literal vs. free translation becomes superfluous, since all of its forms, whether documentary or instrumental, are equally valid procedures, depending on the Skopos. Because of Skopos variation with text receivers, the Skopos of source text and target text may be different. When the Skopos differs, Funktionsanderung (change of function) occurs, thus equivalence is superseded by adequacy. These two goals, adequacy and equivalence (often called 'fidelity' and 'fluency') are not easy to reconcile. The measure of adequacy is the equivalence between the meaning of the original text's item and the meaning of the translated one. Thus, adequacy is the goal and result of the accurate translation, while equivalence is the means of achieving this goal.

According to Txabarriaga (2009), the real indicators of proficiency in translation are knowledge of the subject matter, knowledge of the relevant terminology, the ability to discern meaning in context and transfer it within the target language constraints, i.e., accurately (all meaning has been transferred), precisely (all nuances of the language, tone, intent, style have been preserved in the target language), correctly (grammar, syntax, orthography rules have been observed), completely (no part of the original was omitted and nothing has been added to the target text), and consistently (specific terms, stylistic elements and languagespecific norms have been consistently used throughout).

However, Tuleja et al. (2011), also mention factual reporting-driven adaptations which refer to adjustments resulting from specific linguistic and cultural practices; language-driven adaptations which refer to adjustments resulting from linguistic characteristics such as the presence or absence of the gender category; convention-driven adaptations which refer to adjustments resulting from whether the orientation of a specialized text changes from left-to-right to right-to-left, for example, when translating from English to Arabic, or from left-to-right to top-tobottom when translating from English to Chinese; and culture-driven adaptations which refer to adjustments resulting from the different norms, customs or practices of a given people (Tuleja et al., 2011).

Deficiencies of translation and unsuitable item content constitute the so-called "nuisance factors" that, by Van de Vijver (2003), may affect the measured results and level of comparability of data across cultures (Van de Vijver, 2003). However, 
even when the requirement of translation's accuracy is met, a specialized text written in one language/culture may not necessarily "travel well" across cultures due to differences in meaning and interpretation (Braun, 2003). Considering the TIQA standards, it is worthy of note that they are endowed with universal features, reflected in the codes of ethics and professional standards of translators all over the world. However, they may be further subdivided into the text-oriented and ethical-deontological standards.

\section{First-line TIQA standards}

Within the functionalist approach, C. Nord describes the Skopos rule as "the topranking rule for any translation" which "says that the translational action is determined by its Skopos", i.e. purpose (Nord, 2014). The Skopos rule highlights the importance of the situation in which the text is supposed to circulate, and the addressee who is the intended user of the said translation, making them both instrumental in determining the Skopos and achieving it. Consequently, Nord (2014) also states that it the translation brief that specifies the Skopos; however, concluding that, in case the commissioner is not sufficiently explicit about the Skopos in their brief, it has to be negotiated on (Van de Vijver, 2003). In turn, the fidelity rule, which is considered subordinate to and governed by the Skopos rule, is defined as a principle that requires a translation and the corresponding source text "to bear some kind of relationship", i.e. to possess intertextual coherence (Van de Vijver, 2003). The fidelity rule in translation industry may be applicable either as the overarching demand for accuracy in the translation brief, or as an implied rule, essential to any translator's/interpreter's professional integrity.

These two rules are reflected in the definition of quality as suggested by Mossop (2014): In general, the quality of something is the totality of its characteristics that bear on its ability to satisfy stated and implied needs. With regard to a translation,

- the set of characteristics which make it fit or unfit for its future readers and the use they will make of it.

- the set of characteristics required by an employer, client or translation agency (Mossop, 2014).

The Skopos rule also provides the rationale for the concept of economic viability of any translation quality assurance procedure in given circumstances. Drugan's (2013), research into the approaches to translation quality models in translation industry shows that "the industry is not concerned solely or principally by how to achieve the highest quality levels, but by how to balance various levels of quality with other core requirements" (Drugan, 2013).

Following upon her research of fidelity, Nord (2007), introduced the concept of loyalty to highlight the "responsibility translators have toward their partners in translational interaction" (Nord, 2007). Whereas concepts such as faithfulness or fidelity usually refer to relationships between the texts themselves, loyalty stresses the translator's responsibilities towards people, i.e. not only with regard to clients and users of their translations, but also with regard to the author(s) of the source text. For C. Nord, the Skopos of the target text must be compatible 
with the intentions of the source text author(s). In order to achieve loyalty, the following requirements must be observed:

- the translator's interpretation should be identical with the sender's intention (interpretation);

- the translator should verbalize the sender's intention in such a way that the target text is able to achieve the same function in the target culture as that which the source text achieved in the source culture (text function);

- the target receiver should understand the text world of the translation in the same way as the source receivers understood the text world of the original (cultural distance);

- the effect the translation has on its readers should be the same as the one the source text has or had on its readers (text effect).

This dichotomy of fidelity vs. loyalty plays a pivotal role in delineating the strategy a translator takes to re-tool the specialized source text in accordance with the target audience's expectations. For instance, the documentary translation which consists in respecting the foreign character of the original, expressly showing that the translation is really a translation, requires the fidelity approach. At the same time, the instrumental translation with its objective to present the translation to the recipient as a true original, and respecting all the standards of the target language and culture and containing no foreign elements, clearly manifests the loyalty approach.

Many specialized texts require the documentary translation. Often, these types of translated documents may be considered 'meta-documents' as they give evidence to a reality rooted in the source culture, the source-culture document. On the other hand, in the case of informative documents (or their parts) the main objective is the transfer of information and, therefore, an instrumental strategy can be adopted. These parts of the documents may be adapted to the targetculture standards, the practice which makes it easier for the recipient to process the text (Nord, 2007).

Case study. Taking the spoken mode into account, we're presuming that, on its own, grammatical and lexical correctness of an expression, in our instance a term, cannot guarantee a smooth and uninterrupted discussion via an interpreter. The case is further complicated when an interpreter has to draw on an existing translation of a document where the said translation of the term was first introduced, and which was a recommended reference material by the commissioner. Thus, one meeting veered off into a terminological discussion: 'youth work' (deemed correct as a result of a clarification discussion as it conveys the highly specialized nature of the term compared to the other suggestion) versus 'work with youth' (a misleading one). The experts, who were using the abovementioned translated document (not intended for publication and containing both variants of the term in translation), were not sure if this was a mistake in translation, or the deeper misunderstanding of the core meaning of this concept on the part of the original experts who authored the document in the original language (Abdelaal, 2019; Daniel, 2001; Károly, 2012). 


\section{Second-line TIQA standards}

The standard of accuracy is considered one of the cornerstones of the overall specialized text's quality structure, despite its elusive and fickle nature. The accuracy's preeminent status may be explained by the nature of terms, i.e. metaphorical bricks of the specialized text's quality temple. The terms are monosemantic; however, they are open to a certain variation on the intralingual and interlingual levels. The translation-originating terminological variation is viewed as a contextual equivalence, diversified by collocation rather than the actual sense development.

Lommel (2018), claims that "a quality translation demonstrates accuracy and fluency required for the audience and purpose and complies with all other specifications negotiated between the requester and provider, taking into account both requester goals and end-user needs" (Lommel, 2018). It may be posited that accuracy involves message (which is conceptual rather than verbal domain) and co-exists quite naturally with the categories of "acceptability" and "readability", "linguistic integrity" (terminological consistency) and "fluency".

All of the ISO standards serving the purpose of unifying the quality standards in real-life translation industry settings dwell upon the issue of quality to one or another extent. Thus, ISO 17100:2015(en) Translation services mention several pre-requisites directly bearing upon the standard of accuracy within the clause “5.3.1. Translation":

- compliance with specific domain and client terminology...ensuring terminological consistency during translation;

- semantic accuracy of the target language content;

- lexical cohesion and phraseology;

- compliance with any proprietary and/or client style guide (including domain, language register, and language variants).

Annex B3 to the above-mentioned ISO 17100:2015(en) Translation services mentions that accuracy is assured by the following post hoc procedures: check (self-revision by translator), revision (by another pair of eyes), review (if requested), proofreading and final verification. Prior to an actual translation, the accuracy is reflected in the standard and optional linguistic specifications. Any viable translation project specifications should encompass "accuracy (including terminology) and fluency (e.g. syntax, spelling, and lexical cohesion); compliance with locale conventions and additional linguistic specification when relevant (e.g. language register)".

The ASTM F2575-14, Standard Guide for Quality Assurance in Translation views specialized text rendering within the context of various government practices and calls for "communicating an identical, uniform message to many locales at the same time, that is, not altering the message even slightly for individual locales, and, in information gathering, retaining the original flavor of the source text, purposely avoiding localization", i.e. accuracy is regarded as a standard devoid of any culture-specific features (Borris \& Zecho, 2018; Duizenberg, 2020). 
In the Interagency Language Roundtable (ILR) Skill Level Descriptions for Interpreter Performance, accuracy is given a preeminent function as it makes for a successful interpretation, i.e. "one that faithfully and accurately conveys the meaning of the source language orally, reflecting the style, register, and cultural context of the source message, without omissions, additions or embellishments on the part of the interpreter" (Interagency Language Roundtable (ILR) Skill Level..., 2007). On a more specific note, Level 5 (Master Professional Performance) requires that an expert interpreter should "provide accurate renditions of informal, formal, and highly formal discourse; convey the meaning of the speaker faithfully and accurately, including all details and nuances".

As to the interpreting activities, ISO 18841:2018 (en) Interpreting services General requirements and recommendations in its Section 4 - Basic Principles claims that faithful interpretation is performed without additions, alterations or omissions affecting its content, style, intent and purpose. In Section 6 Qualifications, it requires to accurately interpret for speakers with different educational and cultural backgrounds (linguistic qualifications); to be self-aware to reduce possible cultural biases (intercultural qualifications).

Another approach to the standard of accuracy in translation is borrowed from exact sciences; this approach differentiates between "accuracy" and "precision". Accuracy refers to how close measurements are to the "true" value, while precision refers to how close measurements are to each other (ISO 57251:1994(en) Accuracy (trueness and precision) of measurement methods..., 1994). If extrapolated on the translation study field, it may be interpreted in the following manner: accuracy demonstrates fidelity to the message, while precision - to the direct lexical correspondence. The two main components of precision are repeatability and reproducibility; thus, precision is contributing to the terminological consistency of the translated specialized text. However, accuracy is interchangeably used with validity, the property substantiating the hypothetical assumptions about the translation quality. We may also describe precision as a localized TIQA criterion, while accuracy has a generalized nature.

Darwish (2012), views the "accuracy/precision" dichotomy as a semantic field concept determining the choice of translation equivalent. Thus, accuracy represents the rendition of source language meaning within the spectrum of semantic field's core-adjacent options (the scattergun effect), while precision corresponds to the rendition of source language meaning in full dimensions, i.e. semantic field's peripheral options (the bull's eye effect) (Darwish, 2012) (Figure $1)$. 


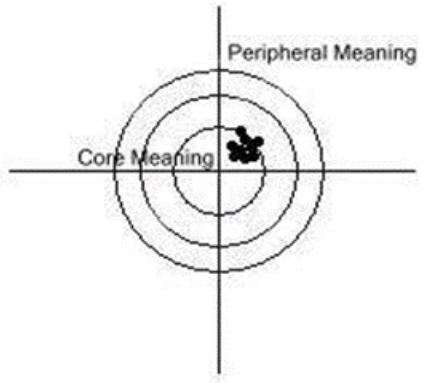

TRANSLATION PRECISION

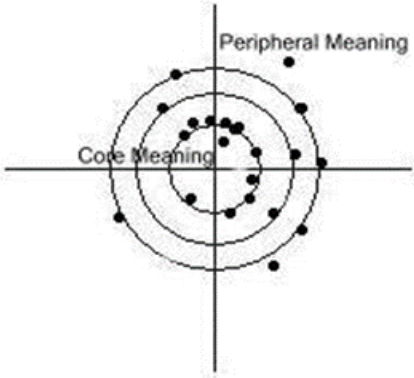

TRANSLATION ACCURACY

Figure 1. Target lexemes approaching the nucleus of semantic field

The categories of accuracy and precision explain the inner workings of gravitational pull in translation. The semasiological salience of units (accuracy) creates a certain 'pull', influencing translator's choices. Thus, highly salient linguistic items (lexis or grammatical constructions) would be more likely to be chosen by translators and thus to be overrepresented in translational corpus data. According to S. Halverston's study, the source units effect the pull, while the target units have magnetism, i.e. the target language salience, represented by precision (Halverson, 2007).

Seen from this perspective of interacting though oppositely directed forces, the quality of translated specialized text may be evaluated and scored. For instance, Nababan (2004), created the following system of scores associated with accuracy indicators (Table 1):

Table 1

Accuracy indicators and corresponding scores

\begin{tabular}{ll}
\hline Score & Accuracy Indicators \\
\hline 1 & The content of the ST is conveyed accurately in the TT. The \\
sentences can be understood clearly. Rewriting is not needed. \\
The content of ST is conveyed accurately to the TT. The sentences \\
can be understood clearly, but the peripheral semes are chosen \\
poorly (precision is violated); thus, the text is not up to the TL \\
conventions of usage. Replacing the words with the suitable ones is \\
needed. \\
The content of the ST is not conveyed accurately in the TT. \\
Restructuring and rewriting whole phrases are needed. \\
ST sentences are not translated into TT. Fragments are \\
unintentionally omitted.
\end{tabular}

\section{Post hoc TIQA standards}

Fluency is the most debated TIQA criterion; it is also the one most scholars find difficult to describe. The universal belief seems to be that fluency is instinctively 
perceived by the listener; it is hardly a combination of component linguistic units. We may thus presume that fluency is the fruit of reception rather than production; and being such, this is a TIQA criterion of post hoc evaluation. Fluency is most often juxtaposed with accuracy; and the general opinion seems to tip in favour of the latter. A person, who speaks slowly, making a lot of pauses, is perceived in a more positive light (unless there are numerous mistakes in his/her discourse) than the one who is glib though erroneous: A steady stream of speech which is highly inaccurate in vocabulary, syntax, or pronunciation could be so hard to understand as to violate an essential aspect of fluency being comprehensible. On the other hand, it is possible for the speaker to be halting but accurate. Sentence level grammatical accuracy that violates principles of discourse and appropriateness is also possible, but such language would not be truly accurate in following the communicative rules of the target language (Ebsworth, 1998).

However, in translation (and especially its spoken mode) this natural eloquence, glibness and quick response seem of a paramount importance, easily overriding the exigency of accurate use of words. Interpreting fluency is the ability to keep speaking at a natural speech rate without pausing, repeating, or stopping for extended periods to think of what to say; it comprises three components: correctness of pronunciation, speech rate and prosody (expressiveness).

In the written mode of interlinguistic mediation, the standard of fluency has gained notoriety due to L. Venuti's relegating it to the "illusion of transparency" created by domestication and translator's invisibility, presumably required by an ethnocentric publishing world (Venuti, 1995). If we extrapolate it to the specialized translation context, fluency may be referred to as simplification of scientific concepts, achieved though explanatory or descriptive rendering, homogenization of the specialized target texts.

The contentious nature of fluency criterion is amply represented in the corresponding quality guidelines. For instance, ISO 17100:2015(en) Translation services speak of "translating in accordance with the linguistic conventions of the target language and relevant project specifications", and as it is further demonstrated by the list of those specifications, the conventions of language use go hand in hand with accuracy requirements. To this end, among other specifications it mentions "lexical cohesion and phraseology", "language register and language variants", "locale and any applicable standards", and what's essential, "target audience" (ISO 17100:2015(en) Translation services..., 2015). The scope of recipient knowledge (their literacy) determines the choice of verbal components in translation.

The translation project specifications (B.3) of the same ISO include an attempt to specify the inner fluency mechanisms ascribing them to syntax, spelling and lexical cohesion, though we consider them the building-blocks of accuracy rather than fluency. However, the sensitiveness to cultural aspects of the locale, "retaining the original flavor of the source text", mentioned by the ASTM F257514 Standard Guide for Quality Assurance in Translation is definitely something only fluency is bringing into the mediated interaction. 
In specialized translation, fluency also involves categories of "legibility" and "readability". Legibility refers to the clarity of form, to the way the text is presented physically on paper, and affects all readers regardless of the purpose of reading. It determines the degree of accessibility to the information contained in the text (i.e. typographical features: typefaces, point sizes, margin widths, text width, line spacing, letter spacing (kerning), paper size etc., which facilitate or hamper information retrieval). By contrast, readability refers to the clarity of content (the number of syllables per word, sentence length, sentence complexity, terminological complexity).

According to Bell (1997), fluency (which he calls "acceptability") is propelled by the illocutionary and perlocutionary forces as well as the principle of communicative cooperation (Bell, 1997). It is receiver-oriented to the same extent as accuracy is being ST-anchored. The translation is thus considered quality if easily understood and accepted by the intended recipients, and the extent of fluency may be evaluated and scored.

Table 2

Fluency indicators and corresponding scores

\begin{tabular}{ll}
\hline Score & Fluency Indicators \\
1 & $\begin{array}{l}\text { The TT text is easy to process. The terms used in the TT can be fully } \\
\text { understood by the TL readers. }\end{array}$ \\
2 & $\begin{array}{l}\text { The TT text is easy to process. However, some of the terms used in } \\
\text { the TT cannot be fully understood by the TL readers. }\end{array}$ \\
3 & $\begin{array}{l}\text { The TT text is hard to process. Half the terms (or the salient ones) } \\
\text { used in the TT cannot be understood by the TL readers. }\end{array}$ \\
4 & $\begin{array}{l}\text { The TT text is extremely hard to process. Most terms used in the TT } \\
\text { cannot be understood or are lost in translation. }\end{array}$ \\
\hline
\end{tabular}

In case of consecutive or simultaneous interpreting, the immediacy of direct communication quality assessment by the participants of an event results in amalgamation of the interpreting and the interpreter him/herself. Firstly, the inclusion of "communication" is intentional because non-professionals (as opposed to language experts) do not focus on separate interpreted parts of a conversation, but rather on its overall seamless-ness, or, in terms of TIQA criteria, fluency. Elegance may be considered as an additional mandatory benchmark of evaluating TIQA standards of formal (ceremonial) interpreting. Secondly, the amalgamation of an interpreter/mediator and his/her output is a result of an interpreter's "visibility", meaning actual awareness by the participants of an event of their presence and indispensable assistance in communication. The aforementioned awareness leads to participants' overall reaction to the interpreter's output: an opinion-based assessment that demonstrates if it was easy to communicate through an interpreter. Consequently, auditory satisfaction requires that the interpreter's output be phonetically comfortable, implying that a calm pleasant voice, a quality which was specifically emphasized by Maksymenko (2008), clear articulation, correct pronunciation, accent (preferably neutral for multicultural audiences), etc., might override accuracy/completeness of the original message. 
Fluency also means extra quality with regard to interpreters coping with adverse conditions (e.g. background noises, poor Internet connection on the part of speakers), taking into account "basic non-correctability" (Nord, 1997), inherent in interpreting. When the working environment is not conductive to quality output, the interpreter's ability to fill in awkward pauses or piece together a disjointed statement serves as an indicator of quality performance under strain.

Professional integrity in its broadest sense reflects heavily on the overall perception of translation/interpretation quality. It is defined and regulated by a multitude of diverse codes of ethics and/or conduct; e.g. the Translator's Charter by the International Federation of Translators states: The translator shall respect the legitimate interests of the user by treating as a professional secret any information which may come into his/her possession as a result of the translation entrusted to him/her (FIT, The Translator's Charter, 1963).

However, abidance by such codes does not necessarily reflect on the output quality as such, it can be a deciding factor in its acceptance. This further leads to other questions such as the adherence to translation brief, i.e. what the commissioner wants and what they get, in stark opposition. Yet, professional integrity as an overall standard also covers a number of ethical-deontological criteria, such as confidentiality, impartiality, and respect for communicative autonomy. The research carried out by McDonough Dolmaya (2011), into seventeen professional codes of ethics for translators demonstrates that confidentiality, together with competence, appears to be the only two widelyacknowledged principles (McDonough Dolmaya, 2011).

The confidentiality clause is always implied but may be prescribed explicitly depending on the arrangement. The reasons for it range from patient's privacy to copyright protection, marketing strategies, to name but a few, and occurring in any field of human activity, as exemplified by strict secrecy requirements in the case of the book Inferno by Dan Brown; these tight security requirements surrounding the translation processes of this particular book even inspired a film by French director R. Roinsard (Webster Ayuso, 2020). This is notwithstanding the common occurrence of the privacy requirement in a mundane setting of any meeting behind the closed doors. Hence, confidentiality might also be regarded as an added-value element to translation, the so-called "esoteric ritual" which elevates the status of both the translator's/ interpreter's profession and the information that only certain stakeholders in the translation process are privy to.

In terms of linguistic integrity, the American Translators Association (ATA) promotes it being the core of what translators and interpreters do, ensuring that nothing is omitted, altered or added to the source language. Linguistic integrity encompasses the notion of staying within the limits of one's qualifications and capabilities, meaning that the interpreter must decline any projects that are beyond them. The concept of linguistic integrity also requires that interpreters enhance their capabilities whenever possible through continuing education in language, industry and professional practice (American Translators Association (ATA), 2020). There are other types of integrity bordering on the "textual quality standards": among them, information integrity referring to the ability of retaining the same information in terms of accuracy, correctness, completeness and 
original intentions (both informative and communicative); internal integrity, i.e. the ability to retain textual coherence and cohesion; and internal referential integrity, i.e. the ability to retain consistency of references throughout the text (Darwish, 2012).

The TIQA pyramid hierarchy, though presumably extant, is also called forth by the translation study research needs, as "applicable specifications can include those of the client, of the TSP itself, and of any relevant industry codes, bestpractice guides, or legislation" (ISO 17100:2015(en) Translation services..., 2015). It means that there are no exact rules or regulations prescribing the TT quality parameters (and thus translation study turns out to be descriptive rather than prescriptive). However, our review of the sources promotes an iterative, step-bystep process of the specialized text quality assurance with consideration of a specific mediated communication interaction context.

\section{Conclusion}

A gifted translator/interpreter knows how to reproduce the message and function of the specialized text/discourse in the manner first described by St. Jerome: non verbum e verbo sed sensum de sensu (not word for word but sense for sense). This underlying principle of a faithful translation presupposes mastery of various equivalence types, understanding terminological variation in the multifaceted cultural settings, associations of register with etymology of lexical units. If anything, specialized translation/interpreting should be culturally competent, take into account the recipient's cultural background, beliefs and values, as well as incorporate this knowledge into the mediated TT delivery. The specialized texts frequently present interpretation difficulties stemming from differences in the academic and research cultures of the original and target languages (i.e., "small cultures", interpretative communities or communities of practice). Furthermore, style and formatting may be quite different from what the target recipients are used to in their native countries. Intrusion of particular features of language use (terminology, grammar, etc.), common in communication among professionals, may impede appropriate understanding on the part of the translator/interpreter, as well as the recipient, thus violating the "mediated communication contract".

The TIQA standard framework includes both text-bound (choate) and ethicaldeontological (inchoate) criteria, arising out of the translator's personality or the specific textual features. Some of the criteria form a dichotomy (e.g. accuracy vs. fluency, fidelity vs. loyalty), which is resolved via preference of the mode (oral or spoken variety or translation) or rank-bound textual considerations (e.g. accuracy vs. precision). However, mostly the TIQA criteria exist in a parallel fashion, mirroring one another, and gaining visibility only via application to a specific study case or translation analysis methodology. The choice of the most efficient translation methodology and procedures, as far as the quality assurance is concerned, depends to a large extent upon the background knowledge and cultural awareness of the initiator (commissioner), but relies upon the due diligence, good practice and skills of the translator. Mediation between cultures requires the communication of ideas and information from one cultural context to the other, creating new sense by accretion of the specialized concepts in a new TL environment. 


\section{References}

Abdelaal, N. M. (2019). Subtitling of culture-bound terms: strategies and quality assessment. Heliyon, 5(4), https://doi.org/10.1016/j.heliyon.2019.e01411

Angelelli, C. V. (2004). Medical interpreting and cross-cultural communication. Cambridge University Press.

Bell, R. T. (1997). Translation and Translating: Theory and Practice/Roger Bell. N.-Y.: Longman.

Borris, D. ., \& Zecho, C. . (2018). The linguistic politeness having seen on the current study issue. Linguistics and Culture Review, 2(1), 32-44.

Braun, M. (2003). Errors in comparative survey research: An overview. Crosscultural survey methods, 137-142.

Chen, K. M., Snyder, M., \& Krichbaum, K. (2002). Translation and equivalence: the profile of mood states short form in English and Chinese. International journal of nursing studies, 39(6), 619-624. https://doi.org/10.1016/S00207489(01)00068-2

Coban, F. (2015). Analysis and training of the required abilities and skills in translation in the light of translation models and general theories of translation studies. Procedia-Social and Behavioral Sciences, 197, 707-714. https:/ / doi.org/10.1016/j.sbspro.2015.07.074

Daniel, T. C. (2001). Whither scenic beauty? Visual landscape quality assessment in the $21 \mathrm{st}$ century. Landscape and urban planning, 54(1-4), 267-281. https://doi.org/10.1016/S0169-2046(01)00141-4

Darwish, A. (2012). Forensic translation: an introduction to forensic translation analysis. Writescope Publishers.

Díaz, B., Sebastián-Gallés, N., Erdocia, K., Mueller, J. L., \& Laka, I. (2011). On the cross-linguistic validity of electrophysiological correlates of morphosyntactic processing: A study of case and agreement violations in Basque. Journal of Neurolinguistics, 24(3), 357-373. https://doi.org/10.1016/j.jneuroling.2010.12.003

Dolmaya, J. M. (2011). Moral ambiguity: Some shortcomings of professional codes of ethics for translators. The Journal of Specialised Translation, 15, 28-49.

Dresp-Langley, B. (2009). The communication contract and its ten ground clauses. Journal of Business Ethics, 87(3), 415-436.

Drugan, J. (2013). Quality in professional translation: assessment and improvement (Vol. 9). A\&C Black.

Duizenberg, M. R. . (2020). Linguistics landscape: A cross culture perspective. Linguistics and Culture Review, 4(1), 15-28.

Ebsworth, M. E. (1998). Accuracy vs. Fluency: Which Comes First in ESL Instruction?. ESL Magazine, 1(2), 24-26.

Grunert, K. G., Brunsø, K., \& Bisp, S. (1993). Food-related life style: Development of a cross-culturally valid instrument for market surveillance (pp. 1-41). Århus, Denmark: MAPP.

Guillemin, F., Bombardier, C., \& Beaton, D. (1993). Cross-cultural adaptation of health-related quality of life measures: literature review and proposed guidelines. Journal of clinical epidemiology, 46(12), 1417-1432.

Halverson, S. (2007). Investigating gravitational pull in translation: the case of the English progressive construction. Texts, process and corpora: research inspired 
by Sonja Tirkkonen-Condit. Joensuu: Publications of the Savonlinna School of Translation Studies, 5, 175-195.

Hatim, B., \& Mason, I. (2014). Discourse and the Translator. Routledge.

Hudelson, P., Dao, M. D., Perron, N. J., \& Bischoff, A. (2013). Interpretermediated diabetes consultations: a qualitative analysis of physician communication practices. BMC family practice, 14(1), 1-9.

Károly, A. (2012). Translation competence and translation performance: Lexical, syntactic and textual patterns in student translations of a specialized EU genre. English for Specific Purposes, 31(1), 36-46. https://doi.org/10.1016/j.esp.2011.05.005

Kashgary, A. D. (2011). The paradox of translating the untranslatable: Equivalence vs. non-equivalence in translating from Arabic into English. Journal of King Saud University-Languages and Translation, 23(1), 4757. https://doi.org/10.1016/j.jksult.2010.03.001

Lommel, A. (2018, March). Translation Quality Metrics. In Proceedings of the AMTA 2018 Workshop on The Role of Authoritative Standards in the MT Environment (pp. 69-94).

Maksymenko, OV (2008). Usnyj pereklad: teoriya, vpravy, teksty (franczuz'ka mova) [Interpreting: theory, exercises, texts (French)]. Vinnytsa, Nova knyga Publ.

Malakoff, M. E. (1992). Translation ability: A natural bilingual and metalinguistic skill. In Advances in psychology (Vol. 83, pp. 515-529). North-Holland. https://doi.org/10.1016/S0166-4115(08)61514-9

Mossop, B. (2014). Revising and editing for translators. Routledge.

Nababan, M. R. (2004). Strategi Penelitian Kualitas Terjemahan in Jurnal Linguistik BAHASA.

Nord, C. (1997). Defining translation functions. The translation brief as a guideline for the trainee translation. Iha do Desterro A Journal of English Language, Literatures in English and Cultural Studies, (33), 039-054.

Nord, C. (2007). Function plus loyalty: Ethics in professional translation. Génesis. Revista Científica do ISAG, 6, 7-17.

Nord, C. (2014). Translating as a purposeful activity: Functionalist approaches explained. Routledge.

Pym, A. (2014). Theories of Localization.(Exploring Translation Theories). Video file]. Retrieved, 24.

Rezvani, R., \& Vakilinejad, M. (2014). An evaluation of translation quality assessment course: Voices from instructors. Procedia-Social and Behavioral Sciences, 98, 1563-1571. https://doi.org/10.1016/j.sbspro.2014.03.579

Saule, B., \& Aisulu, N. (2014). Problems of translation theory and practice: original and translated text equivalence. Procedia-Social and Behavioral Sciences, 136, 119-123. https://doi.org/10.1016/j.sbspro.2014.05.300

Seleskovich, D. (2015). Interpreter in international conferences. Language and communication problems. Garnier classics.

Simanjuntak, F. (2019). A study on quality assessment of the translation of an abstract text English idioms errors made by Jordanian EFL undergraduate students by Google Translate. International Journal of Linguistics, Literature and Translation, 2(4), 38-49.

Singleton, K., \& Krause, E. (2009). Understanding cultural and linguistic barriers to health literacy. The Online Journal of Issues in Nursing, 14(3).

Torresi, I. (2020). Translating promotional and advertising texts. Routledge. 
Toury, G. (1995). Descriptive translation studies and beyond (Vol. 4). Amsterdam: J. Benjamins.

Tuleja, E. A., Beamer, L., Shum, C., \& Chan, E. K. (2011). Designing and developing questionnaires for translation tutorial. IEEE transactions on professional communication, 54(4), 392-405.

Txabarriaga, R. (2009). IMIA guide on medical translation. International Medical Interpreters Association. January2009.

UNESCO. (1976). Recommendation on the legal protection of translators and translations and the practical means to improve the status of translators: adopted by the General Conference at its nineteenth session, Nairobi, 22 November 1976. Unesco.

Van de Vijver, F. J. (2003). Bias and equivalence: Cross-cultural perspectives. In Cross-cultural survey methods (pp. 143-155). Wiley.

Venuti, L. (1995). Translation, authorship, copyright. The Translator, 1(1), 1-24.

Webster Ayuso, J. (2020). Literary lockdown: how translating a Dan Brown novel made for a thriller plot. The Guardian. 